\title{
Necesidad de políticas de salud pública en población adulta mayor: indicadores de envejecimiento en un Instituto de Seguridad Social en México
}

\author{
Daniel López-Hernández*, Nadhyieli Orozco-Campos, Irma Luz Riva Palacio Chiang y Sam y \\ Margarita Blanco-Cornejo \\ Subdirección de Prevención y Protección a la Salud, Instituto de Seguridad y Servicios Sociales de los Trabajadores del Estado, Ciudad de México, \\ México
}

\section{Resumen}

Introducción: Un diagnóstico integral de población contempla dentro de sus componentes el análisis demográfico poblacional, que es indispensable en la formulación de las políticas públicas. La política de población tiene una naturaleza claramente transversal, pues todas las acciones en los ámbitos económico, social, político, cultural, geográfico, y obviamente, el demográfico, repercuten de una manera directa o indirecta en ella. Objetivos: Determinar la dinámica poblacional y el crecimiento global de la población adulta mayor (PAM) de 60 años y más. Materiales y métodos: Estudio transversal, retrospectivo. La información se obtuvo de los anuarios estadísticos institucionales del Instituto de Seguridad y Servicios Sociales de los Trabajadores del Estado, México (de 1999-2015). Se analizaron varios indicadores demográficos de envejecimientoDE). Resultados: Se observó un incremento constante en puntos porcentuales en la proporción de PAM, el índice de envejecimiento, la razón de dependencia demográfica de la vejez, índice global de dependencia, índice dependencia de viejos e índice de estructura de la población activa (6, 19.2, 15.5, 8.5, 8.2 y 31.2\%, respectivamente). El indicador global de ancianidad y el índice de masculinidad mostraron una disminución (0.6 y 3.1\%, respectivamente). Conclusiones: Nuestros datos aportan evidencia que sugiere modificar y generar políticas públicas acordes a la PAM.

PALABRAS CLAVE: Dinámica poblacional. Políticas de salud pública. Población adulta mayor. Indicadores de envejecimiento.

\begin{abstract}
Introduction: An integral diagnosis of population contemplates within its components the population demographic analysis that is indispensable in the formulation of public policies. Population policy has a clearly transversal nature, since all actions in the economic, social, political, cultural, geographical, and obviously, demographic fields, have direct or indirect repercussions on it. Objectives: To determine the population dynamics and the global growth of the older adult population (OAP) of 60 years and more. Materials and methods: Cross-sectional, retrospective study. The information was obtained from the statistical yearbooks of the institute of security and social services of state workers, Mexico (1999-2015). Several demographic ageing indicators were analyzed. Results: There was a constant increase in percentage points in the proportion of OAP, index of ageing, demographic dependency ratio of old age, global index of dependence, index of dependence of old people, and index of the active population structure $(6,19.2,15.5,8.5,8.2$ and $31.2 \%$, respectively). The indicator global index of dependence and masculinity showed a decrease (0.6 and $3.1 \%$, respectively). Conclusions: Our data provide evidence that suggests modifying and generating public policies according to OAP.
\end{abstract}

KEY WORDS: Population dynamics. Public health policies. Elderly population. Indicators of ageing.

Correspondencia:

*Daniel López Hernández

E-mail: daniel16042016@gmail.com
Fecha de recepción: 06-03-2019

Fecha de aceptación: 19-03-2019

DOI: $10.24875 / G M M .19005140$
Gac Med Mex. 2019;155(Suppl 1):S10-S15

Disponible en PubMed www.gacetamedicademexico.com 


\section{Introducción}

A nivel mundial se observa una transición epidemiológica de la población adulta mayor (PAM) ${ }^{1,2}$ que constituye un reto para la sociedad y los sistemas de salud, en materia de asistencia médica, y en particular para la asignación del presupuesto destinado a los servicios de salud; los cuales deberán responder de forma apropiada al envejecimiento de la población. Asimismo, genera retos para diseñar y desarrollar nuevas políticas públicas, con base en los factores asociados con la población de cada país ${ }^{2-4}$. No obstante, aunque la Organización Mundial de la Salud propone estrategias de acción generales para lograr un envejecimiento activo, los planes de políticas públicas se deben basar en las características sociodemográficas de cada población. Para ello, es importante conocer la dinámica poblacional, que es una medida demográfica fundamental para adoptar políticas claves, con base en datos reales, y así generar cambios sistémicos y lograr un envejecimiento activo y saludable, dando como resultado una respuesta integral de salud pública. En este contexto, la fracción XII del Artículo 34 de la Ley del Instituto de Seguridad y Servicios Sociales de los Trabajadores del Estado (ISSSTE), contempla que, como parte de los servicios de atención médica preventiva tendientes a proteger la salud de los derechohabientes, el Instituto incluirá la atención del envejecimiento. Sin embargo, el Manual de Organización General del ISSSTE, dentro de su estructura orgánica, no incluye ninguna Jefatura de Servicios o de Departamento de envejecimiento saludable, acotando las acciones a la atención médica curativa y de rehabilitación vinculada a los módulos gerontológicos instaurados dentro de las unidades del primer nivel de atención. El objetivo del presente estudio es determinar el perfil de la dinámica poblacional y el crecimiento de la población de 60 años y más, en el ISSSTE, y con base en ello analizar las implicaciones que esto conlleva para las políticas de salud pública.

\section{Métodos}

Se realizó un estudio transversal, retrospectivo, con arreglo a las buenas prácticas clínicas de nuestra legislación, y de la Declaración de Helsinki, que incluyó la población amparada del ISSSTE. La información se obtuvo de los anuarios estadísticos de un periodo de 17 años (1999-2015), que no incluyen datos de pacientes. La PAM se definió como las personas de 60 y más años. Se calcularon los siguientes indicadores demográficos de envejecimiento: la proporción $(\mathrm{P})$ de la PAM, la tasa de crecimiento de la PAM y varios índices de envejecimiento (razón de dependencia demográfica de la vejez, de masculinidad, generacional de ancianidad, global de dependencia, de dependencia de viejos, de la estructura de la población activa, de Friz [IFriz], de Sundbarg [ISundbarg], de Burgdöfer [IBurgdöfer] y de Sauvy [ISauvy]), con base en las fórmulas y criterios reportados por la Secretaria de Salud y Torres-Degró ${ }^{5,6}$. De acuerdo al IFriz, el ISundbarg y el IBurgdöfer se clasificó a la población en tres categorías: población joven, madura y vieja ${ }^{6}$. Todas las variables cuantitativas se describen como media aritmética, desviación estándar (DE), mínimo (mín), máximo (máx), mediana $(\mathrm{md})$, rango ( $\mathrm{r}$ ) y rango intercuartílico (RIC). Para la pirámide de población, se calculó el porcentaje de personas por grupos quinquenales y sexo. Las proporciones se compararon mediante la prueba $Y$ cuadrada. Los indicadores de envejecimiento por sexo se compararon con la prueba de comparación de medianas para muestras independientes. Los valores de $p$ $<0.05$ (pruebas de 2 colas) se consideraron significativos. Los datos se analizaron con el paquete estadístico SPSS ${ }^{\circledR}$ para Windows ${ }^{\circledR}$ versión 21.

\section{Resultados}

La población amparada pasó de 9,896,695 (1999) a $12,973,731$ (2015) personas (incremento de 3,077,036 individuos). Del total de derechohabientes, el $19.4 \%$ (hombres: 9.3\%, mujeres: 10.1\%) (Fig. 1) corresponde a la PAM (crecimiento de 1,190,365 individuos, correspondiente al $6 \%$ ). A su vez, de este grupo etario $(n=2,511,665)$, el $48 \%(n=1,207,300)$ son hombres y el $52 \%(n=1,304,365)$ son mujeres. Se observó un incremento y una disminución significativos en la P-PAM (1999: $13.4 \%$ vs. 2015: 19.4\%; $p<0.0001$ ) y de los grupos poblacionales de entre 0-14 (1999: $35.6 \%$ vs. 2015 : $34.2 \% ; p<0.0001)$ y de $15-59$ años (1999: $51.1 \%$ vs. 2015 : $46.5 \%$; $p<0.0001$ ), respectivamente. El cambio de la pirámide poblacional muestra un ensanchamiento en los grupos etarios correspondientes a la punta y una estrechez muy notable en el centro y poco perceptible en su base (Fig. 1). La tasa de crecimiento de la PAM se incrementó significativamente, de 1 a 9 personas por cada 100 derechohabientes (2000-2005: 1, 2005-2010: 2.4 y 2010-2015: 9.1). El crecimiento porcentual promedio de la población, en 17 años, es del 1.68\% (DE: 0.74; mín: 0.41; máx: 3.37; 
Tabla 1. Perfil histórico de los indicadores demográficos de envejecimiento del Instituto de Seguridad y Servicios Sociales de los Trabajadores del Estado, del periodo de 1999 a 2015

\begin{tabular}{|l|c|c|c|c|c|c|c|}
\hline Índices & 1999 & $\mathbf{2 0 0 0}$ & $\mathbf{2 0 0 1}$ & $\mathbf{2 0 0 2}$ & $\mathbf{2 0 0 3}$ & $\mathbf{2 0 0 4}$ & $\mathbf{2 0 0 5}$ \\
\hline PAM 60 y más años & $8,575,395$ & $8,722,188$ & $8,870,250$ & $8,933,708$ & $8,971,039$ & $9,066,869$ & $9,193,650$ \\
\hline Población < 60 años & $1,321,300$ & $1,343,673$ & $1,366,273$ & $1,375,781$ & $1,381,279$ & $1,395,834$ & $1,414,559$ \\
\hline Proporción de PAM 60 y más años (\%) & 13.35 & 13.35 & 13.35 & 13.34 & 13.34 & 13.34 & 13.33 \\
\hline Índice de Vejez (\%) & 37.52 & 37.55 & 37.6 & 37.66 & 37.74 & 37.81 & 37.88 \\
\hline Razón de dependencia de la vejez (\%) & 26.14 & 26.12 & 26.09 & 26.05 & 26.01 & 25.97 & 25.91 \\
\hline Generacional de la ancianidad (\%) & 2.74 & 2.74 & 2.75 & 2.76 & 2.76 & 2.77 & 2.78 \\
\hline Global de dependencia (\%) & 83.94 & 83.82 & 83.65 & 83.4 & 83.12 & 82.87 & 82.6 \\
\hline Dependencia de viejos (\%) & 18.49 & 18.47 & 18.45 & 18.42 & 18.38 & 18.35 & 18.31 \\
\hline Estructura de la población activa (\%) & 60.81 & 60.85 & 60.91 & 61 & 61.11 & 61.2 & 61.29 \\
\hline PAM: población adulta mayor. & & & & & &
\end{tabular}
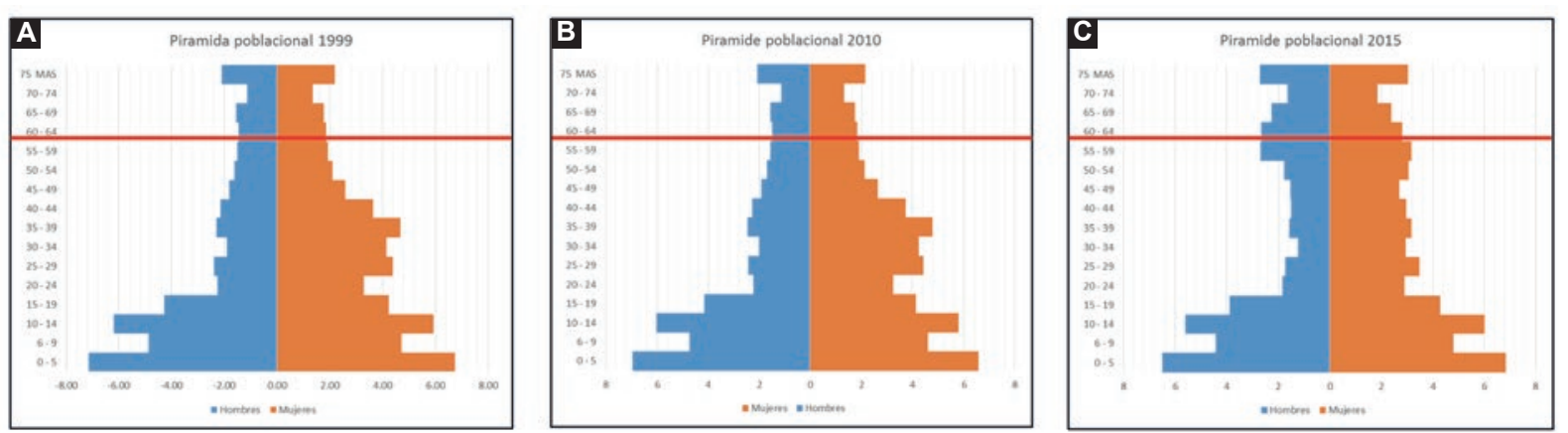

Figura 1. A: Población de 1999; B: Población de 2010; C: Población de 2015. Pirámides de población del Instituto de Seguridad y Servicios Sociales de los Trabajadores del Estado.

r: 2.95; md: 1.66; RIC: 1.34-1.81). En términos absolutos, se observa un crecimiento promedio de 192,315 derechohabientes (DE: 89,782; mín: 42,829; máx: 403,871; r: 361,042; md: 177,104; RIC: 163,251220,752). Los valores promedio para los indicadores de envejecimiento son: P-PAM 14.33 (md: 13.3; RIC: 13.35-13.32); índice de envejecimiento 41.0 (md: 38.1; RIC: 38.4-37.7); índice de razón de dependencia demográfica de la vejez 28.5 (md: 25.97; RIC: 26.1325.69); índice global de dependencia 84.0 (md: 82.87; RIC: 83.88-81.37); índice de dependencia de viejos 19.7 (md: 18.35; RIC: 18.48-18.14), índice de la estructura de la población activa 66.8 (md: 61.58; RIC: 61.95-61.06), índice generacional de ancianidad 2.7 (md: 2.77; RIC: 2.82-2.74) e índice de masculinidad 80.2 (md: 80.66; RIC: 80.95-80.45). Al comparar los datos de 1999 y 2015, se observó un incremento significativo en todos los indicadores (P-PAM: 6\%; índice de envejecimiento: $19.2 \%$; índice de razón de dependencia demográfica de la vejez: $15.5 \%$; índice global de dependencia: $8.5 \%$; índice de dependencia de viejos: $8.2 \%$ e índice de la estructura de la población activa: 31.2\%); a excepción del índice generacional de ancianidad y del índice de masculinidad, que mostraron una disminución significativa (0.6 y 3.1\%, respectivamente). Al analizar cada indicador, los dos indicadores que muestran un incremento constante son el índice de envejecimiento y el índice de la estructura de la población activa; el indicador que mostró un incremento paulatino y un descenso posterior, a partir del año 2013, fue el índice generacional de ancianidad. Así también, la P-PAM, y los índices de razón de dependencia demográfica de la vejez, global de dependencia y de dependencia de viejos mostraron una disminución paulatina en el periodo de 19992012, y a partir del año 2013, un incremento constante en todos ellos (Tabla 1). De acuerdo con los valores obtenidos por el IFriz nuestra población corresponde con una población joven, consistente con el ISundbarg, pero los resultados del IBurgdöfer indican un cambio poblacional, de una población joven a una madura, mientras que el ISauvy sugiere una población vieja (índices mayores de 30\%). El análisis por sexo muestra los mismos patrones, a excepción del índice 


\begin{tabular}{|c|c|c|c|c|c|c|c|c|c|c|}
\hline $\mathbf{2 0 0 6}$ & $\mathbf{2 0 0 7}$ & $\mathbf{2 0 0 8}$ & $\mathbf{2 0 0 9}$ & $\mathbf{2 0 1 0}$ & $\mathbf{2 0 1 1}$ & $\mathbf{2 0 1 2}$ & $\mathbf{2 0 1 3}$ & $\mathbf{2 0 1 4}$ & $\mathbf{2 0 1 5}$ \\
\hline $\mathbf{9 , 3 5 9 , 0 2 3}$ & $9,518,045$ & $9,795,683$ & $10,046,672$ & $10,397,451$ & $10,583,333$ & $10,794,878$ & $10,276,780$ & $10,372,518$ & $10,462,066$ \\
\hline $1,439,925$ & $1,462,886$ & $1,505,061$ & $1,542,811$ & $1,595,903$ & $1,623,397$ & $1,654,731$ & $2,353,789$ & $2,431,299$ & $2,511,665$ \\
\hline 13.33 & 13.32 & 13.32 & 13.31 & 13.31 & 13.3 & 13.29 & 18.64 & 18.99 & 19.36 \\
\hline 37.9 & 38.09 & 38.16 & 38.24 & 38.33 & 38.35 & 38.36 & 54.11 & 55.32 & 56.66 \\
\hline 25.9 & 25.77 & 25.72 & 25.66 & 25.6 & 25.56 & 25.53 & 39.71 & 40.67 & 41.66 \\
\hline 2.78 & 2.81 & 2.82 & 2.83 & 2.84 & 2.85 & 2.85 & 2.23 & 2.19 & 2.14 \\
\hline 82.53 & 81.79 & 81.52 & 81.21 & 80.87 & 80.82 & 80.6 & 91.47 & 91.99 & 92.41 \\
\hline 18.3 & 18.2 & 18.17 & 18.12 & 18.08 & 18.05 & 18.03 & 25.53 & 26.09 & 26.67 \\
\hline 61.32 & 61.58 & 61.67 & 61.78 & 61.9 & 61.94 & 61.96 & 91.71 & 91.89 & 92.03 \\
\hline
\end{tabular}

de la estructura de la población activa, el cual en la población de hombres mostró un incremento en el año 2013. Además, la P-PAM, el índice de razón de dependencia demográfica de la vejez, el global de dependencia, el de dependencia de viejos y el de la estructura de la población activa son significativamente más elevados en hombres que en las mujeres; mientras que los valores del índice de envejecimiento y generacional de ancianidad son significativamente más elevados en las mujeres (Tabla 2).

\section{Discusión}

Se estima que, en 2025, la P-PAM proyectada para la región de las Américas será similar a la proyección para México en el año 20307. Sin embargo, actualmente esta proporción es menor a nuestra población de estudio, lo cual representa un cambio demográfico importante y una realidad ineludible. En cuanto a la tasa de crecimiento de nuestra población, se observó un incremento significativo, el cual es mayor a la esperada a nivel nacional ${ }^{5}$. Estos datos representan un reto importante para la institución y su patrimonio social, puesto que cada vez un mayor número de personas requieren: atención especializada en salud, acceso al sistema de pensiones y protección social, inclusión en las estrategias de promoción y prevención de enfermedades, participación en programas de empoderamiento, grupos de ayuda mutua y, actividades de esparcimiento que favorezcan un envejecimiento activo y saludable ${ }^{8}$; por ende, políticas incluyentes para este grupo poblacional. Nuestros datos indican que las necesidades potenciales de apoyo social destinadas a la población serán mayores que para otras instituciones del país ${ }^{9}$, que los apoyos de asistencia social para la población derechohabiente será un factor muy importante a considerar en el desarrollo de políticas públicas e incluso que las políticas deberán considerar las diferencias respecto del sexo (Tabla 2). Estas diferencias son un reflejo del mayor número de mujeres en edad laboral $(2,306,634$ [38\%] vs. 3,722,258 [62\%], de hombres y mujeres de 15 a 59 años, respectivamente) y una proporción más homogénea de $\operatorname{PAM}(1,207,300$ [48\%] vs. 1,304,365 [52\%] de hombres y mujeres, respectivamente). Otro aspecto muy importante para considerar son los cuidadores de la PAM de 65 y más años. Actualmente se tienen programas que contemplan asistencia social, como visitas domiciliarias, sin embargo, la población encargada del cuidado de este grupo etario es una población vulnerable de colapso, por las actividades inherentes y el cambio del estilo de vida que representa. El índice generacional de ancianidad ha ido aumentando lentamente desde 1999, a diferencia de Puerto Rico, que ha disminuido ${ }^{6}$. En consecuencia, nuestros datos proporcionan evidencia que sugiere la generación de programas dirigidos a la prevención del colapso del cuidador. El índice global de dependencia (población total: 92/100, hombres: $113 / 100$ y mujeres: 78/100 personas económicamente activas) y el índice de dependencia de viejos (población total: 26/100, hombres: 32/100 y mujeres: 22/100 personas en edad laboral), indican que hay más hombres dependientes de personas económicamente activas y en edad laboral, en comparación a las mujeres, lo que sugiere que la población de hombres podría ser más susceptible de requerir apoyos de asistencia social y cuidados por parte de los familiares directos como esposa, hijos y hermanos; 
Tabla 2. Comparación de los indicadores de vejez por sexo

\begin{tabular}{|l|c|c|c|c|}
\hline Indicador & $\begin{array}{c}\text { Población total } \\
n=17 \\
\text { Md (RI) }\end{array}$ & $\begin{array}{c}\text { Hombres } \\
n=17 \\
\text { Md (RI) }\end{array}$ & $\begin{array}{c}\text { Mujeres } \\
n=17 \\
\text { Md (RI) }\end{array}$ \\
\hline PPAM & $13,34(13,35-13,32)$ & $13,97(13,97-13,96)$ & $12,83(12,86-12,78)$ & 0,001 \\
\hline IE & $38,09(38,36-37,70)$ & $34,91(35,25-34,41)$ & $41,41(41,61-41,15)$ & 0,001 \\
\hline RDDV & $25,97(26,13-25,69)$ & $30,26(30,66-30,89)$ & $22,66(23,03-22,89)$ & 0,001 \\
\hline IGA & $2,77(2,82-2,74)$ & $2,31(2,37-2,27)$ & $3,19(3,24-3,16)$ & $<0,001$ \\
\hline IGD & $82,87(83,88-81,37)$ & $104,59(106,22-102,11)$ & $68,44(69,13-67,45)$ & $<0,001$ \\
\hline IDV & $18,35(18,48-18,14)$ & $21,84(22,03-21,54)$ & $16,03(16,13-15,87)$ & 0,001 \\
\hline IEPA & $61,58(61,95-61,06)$ & $66,48(67,18-65,52)$ & $58,45(58,61-58,23)$ & 0,001 \\
\hline
\end{tabular}

Md: mediana; RI: rango intercuartílico; PPAM: proporción de población adulta mayor; IE: índice de envejecimiento; RDDV: razón de dependencia demográfica de la vejez; IGA: índice generacional de ancianidad; IGD: índice global de dependencia; IDV: índice de dependencia de viejos; IEPA: índice de la estructura de la población activa.

pero en nuestro medio, el mayor porcentaje de cuidadores primarios (datos no publicados) y la mayor proporción de personas en edad laboral son mujeres, por tanto, se esperaría una mayor prevalencia de colapso del cuidador en este grupo poblacional. Esto implica incorporar en los programas educativos acciones de empoderamiento dirigidos a la población como una política de salud pública prioritaria en medicina preventiva. Dado que la población de 15 a 64 años es considerada la estructura de la población activa hábil para laboral ${ }^{6}$, es importante conocer el grado de envejecimiento de este sector, no solo por sus implicaciones económicas, sociales y culturales, sino también por su trascendencia en la generación de políticas de salud pública, ya que este grupo de población demanda una dinámica diferente para los sistemas de salud. Nuestros hallazgos indican que, aunque podemos considerar que la estructura de la población laboral es joven, ya hay una transición a una población laboral vieja, lo que incrementará la demanda al sistema de pensiones y protección social del Instituto y la demanda de atención para la PAM, que será mayor en los próximos años. Además, podemos inferir que se incrementará con ello la prevalencia de las enfermedades propias de este grupo poblacional $y$, en consecuencia, el gasto para el sistema de salud. Por lo tanto, nuestros hallazgos aportan evidencia para considerar la inclusión de programas de inversión financiera en los servicios de salud dirigidos a esta población, como una política pública factible. A este respecto, se ha demostrado que el ahorro a los sistemas de salud por cada dólar invertido en programas de promoción a la salud es una política viable 9 . Aunque nuestros datos solo incluyen información del ISSSTE, tienen representatividad nacional para la población derechohabiente. Además, el periodo de análisis es un periodo amplio, lo que permitió observar la dinámica poblacional de cada indicador y ampliar las perspectivas de aplicación de nuestros hallazgos. Nuestros datos muestran que la política de salud debe contemplar como medidas prioritarias e inmediatas: mejorar el acceso a la medicina preventiva, adecuar los servicios de salud a la PAM, contemplar las diferencias de género, ser incluyentes de la PAM y de la población en edad laboral; considerar la dinámica poblacional, garantizar el abasto de medicamentos esenciales, dotar de una infraestructura adecuada a los servicios de atención primaria, incluir una mayor incorporación de recursos materiales, financieros y humanos en materia de geriatría, incorporar programas ad hoc a la población e incluir un modelo de intervención integral para un envejecimiento activo y saludable. Abordar este tipo de medidas como una política de salud pública prioritaria es esencial para generar una atención integral a la población y mejorar los gastos en salud, para responder al desafío poblacional futuro. En este contexto, falta construir un marco legal con mecanismos de seguridad y protección social que sirvan para combatir de forma clara y expedita la pobreza, la vulnerabilidad, la exclusión social, y que aseguren la generación de pisos nacionales de protección social que sean inclusivos y no discriminatorios, para este grupo de población, así como definir y delimitar los criterios a fin de evaluar la idoneidad para asegurar una vida digna e incorporar la participación de las personas que trabajan en la economía informal, de los grupos desfavorecidos y de las personas con necesidades especiales; definir y proporcionar garantías básicas de seguridad social a la población residente del país, bajo principios de universalidad de la protección, basada en la solidaridad social, el derecho a las prestaciones prescrito por un marco legislativo; que 
incluya el respeto de los derechos y la dignidad de las personas, la solidaridad en la financiación, asociada a la búsqueda de un equilibrio óptimo entre las responsabilidades y los intereses de aquellos que financian y se benefician de los regímenes de seguridad social; con inclusión de los mecanismos de financiación y los sistemas de prestaciones; gestión financiera y administración sanas, responsables y transparentes; sostenibilidad financiera, fiscal y económica, teniendo debidamente en cuenta la justicia social y la equidad; coherencia con las políticas sociales, económicas, de salud y de empleo; coherencia entre las instituciones encargadas de la prestación de servicios de protección social; servicios públicos de alta calidad que mejoren el desempeño de los sistemas de seguridad social; eficacia y accesibilidad de los procedimientos de reclamación y de recurso; seguimiento regular de la aplicación y evaluación periódica; pleno respeto de la negociación colectiva y de la libertad sindical para todos los trabajadores, y participación tripartita con las organizaciones representativas de los empleadores y de los trabajadores, así como la celebración de consultas con otras organizaciones pertinentes y representativas de personas interesadas ${ }^{10}$.

\section{Conclusión}

Nuestros datos aportan evidencia que sugiere la incorporación de estrategias y mecanismos de seguridad y protección social acordes a la dinámica poblacional basada en el empleo de los indicadores demográficos de envejecimiento y el crecimiento de la PAM; con la finalidad de fortalecer la atención primaria de salud en el sistema público para este grupo poblacional.

\section{Conflicto de intereses}

Los autores declaran no tener ningún conflicto de intereses.

\section{Bibliografía}

1. Temas de Salud. Envejecimiento [Internet]. Ginebra: Organización Mundial de la Salud [10 ago 2017]. Disponible en: https://www.who.int/topics/ ageing/es/.

2. Organización Mundial de la Salud. Informe mundial sobre el envejecimiento y la salud. EE.UU.; 2015.

3. Bloom DE. 7 billion and counting. Science. 2011;333:562-9

4. Christensen K, Doblhammer G, Rau R, Vaupel JW. Ageing populations: the challenges ahead. Lancet. 2009;374:1196-208.

5. Perfil epidemiológico del adulto mayor en México 2010. México: Secretaría de Salud, Subsecretaría de Prevención y Promoción de la Salud, Dirección General de Epidemiología; 2011.

6. Torres-Degró A. Envejecimiento demográfico: un acercamiento a los métodos cuantitativos. CIDE digital [Internet]. 13 ago 2017 [citado 11 ago 2017];1(2):79-02. Disponible en: https://revistas.upr.edu/index.php/ cidedigital/article/view/11864

7. Crecimiento acelerado de la población adulta de 60 años y más de edad: Reto para la salud pública [Internet]. Organización Panamericana de la Salud; 2012. Disponible en: https://www.paho.org/hq/index.php?option=com_content\&view=article\&id=2796:2010-crecimiento-acelerado-poblacion-adulta-60-anos-mas-edad-reto-salud-publica\&ltemid=1914\&lan$\mathrm{g}=\mathrm{es}$.

8. Secretaría de Desarrollo Social. Subsecretaría de Prospectiva, Planeación y Evaluación I. Avances y retos de la política social. Índice de envejecimiento a nivel global y en México. Indicadores de Desarrollo Social. 2013;2(63).

9. Banke-Thomas AO, Madaj B, Charles A, van den Broek N. Social Return on Investment (SROI) methodology to account for value for money of public health interventions: a systematic review. BMC Public Health. 2015;15:582.

10. Recomendación sobre los pisos de protección social, 2012 (núm. 202). Ginebra: Organización Internacional del Trabajo, 101ª reunión CIT (14 junio 2012); 2012. 